\title{
Autoimmune pancreatitis: a case of difficult diagnosis
} \author{
Carlo Boselli ${ }^{3}$, Giuseppe Noya ${ }^{3}$ \\ ${ }^{1}$ Department of General Surgery, University of Perugia, St. Maria Hospital, Terni, Italy \\ 2Department of Surgical Sciences, Sapienza University of Rome, Rome, Italy \\ ${ }^{3}$ Department of General and Oncologic Surgery, University of Perugia, Perugia, Italy
}

Roberto Cirocchi ${ }^{1}$, Alberto Santoro ${ }^{2}$, Alessia Corsi ${ }^{1}$, Paolo Ronca ${ }^{1}$, Jacopo Desiderio ${ }^{1}$, Francesco Barberini ${ }^{3}$,

Prz Gastroenterol 2015; 10 (1): 51-53

DOI: $10.5114 / p g .2014 .47500$

Key words: pancreatitis, autoimmune, diagnosis.

Address for correspondence: Alessia Corsi MD, Department of General Surgery, University of Perugia, St. Maria Hospital, Via Tristano di Joannuccio, Terni, 05100, Italy, e-mail: alessia.cor@libero.it

\begin{abstract}
Autoimmune pancreatitis (AIP) is an insidious disease of non-specific symptomatology. To make correct diagnosis three different findings must correlate: radiological imaging, serological markers, and histology. This is not easy, and furthermore an incorrect diagnosis can lead to incorrect management and even patient death. We present our experience with a case of AIP in a young woman (34 years old) affected by different autoimmune pathologies with a history of abdominal pain. The diagnosis was made correlating histological findings and anamnestic data, although there were no radiological or serological findings. However, the management of this case was complicated by acute pancreatitis. In our case, we had only a histological sample and anamnestic data. So in these cases of positive history for autoimmune disorders and unclear clinical signs, AIP should be considered in differential diagnosis.
\end{abstract}

\section{Introduction}

Idiopathic pancreatitis accounts for between $30 \%$ and $40 \%$ of all chronic pancreatitis [1]. In 1961 Sarles et al. described an association between some forms of chronic pancreatitis and the finding of a hyperglobulinaemia [2]. It was assumed that there was an autoimmune component as a possible aetiological factor, so a new nosological entity was set up, even if not universally accepted, and defined as autoimmune chronic pancreatitis $[1,2]$. Also, in the literature several cases of wrong diagnosis are described; this has led to pancreatic resection because of pancreatic lesions produced by this condition, similar to cancer lesions [3, 4].

Patients with autoimmune pancreatitis (AIP) have these kinds of clinical manifestations: some are asymptomatic, others report vague abdominal pain, may have jaundice, and sometimes acute pancreatitis [5] with or without association of other autoimmune diseases [1].

Although this disease has a worldwide spread, an increase in incidence has been observed especially in Japan [6], enough to induce the "Japanese Pancreatic Society" in 2002 to publish new guidelines for the correct diagnosis, and then further expanded $[7,8]$.
These criteria are based on interpolation of three diagnostic lines: radiological imaging, serological markers, and histology $[7,8]$.

The Mayo Clinic suggests, for the diagnosis of autoimmune pancreatitis, its HISORT criteria, which also associate with previous involvement of other organs and the response to steroid therapy (Histology, the pancreatic Imaging, Serology - IgG4 > 140 mg\%, Other organ involvement, including biliary strictures, parotid/lacrimal gland involvement, mediastinal lymphadenopathy, retroperitoneal fibrosis Response to steroid treatment resolution/marked improvement of pancreatic and extrapancreatic manifestations) [9]. We present a clinical case that underlines the difficulties in making correct diagnosis and consequently complex management of the patient.

\section{Case report}

The patient, a woman of 34 years, affected by autoimmune diseases (uveitis, which over the years has resulted in the loss of visual acuity, diabetes mellitus type I, thyroiditis) reported for 2 years, a history of recurrent abdominal pain in the epigastrium and right hypochon- 
drium. She had been subjected to numerous investigations and admissions, but they had not revealed the cause of these symptoms.

The patient had recently undergone plastic wall laparotomy (sutures of the fascia) in emergency for epigastric hernia and had constant abdominal pain. During the hernioplasty there was the occasional finding of increased consistency of the pancreatic head. This finding pushed clinicians to refer the patient for other diagnostic tests (computed tomography abdomen, esophagogastroduodenoscopy and abdominal magnetic resonance imaging) to clarify its nature (Figure 1).

For the sudden onset of continuous pain in the right hypochondrium and epigastrium the patient was admitted to the emergency at our unit and was diagnosed with acute cholecystitis (thickening of the wall of the gallbladder ultrasound, fever, leucocytosis, but normal amylase).

The patient underwent emergency surgery and cholecystectomy was performed, and then was taken an incisional biopsy of the lesion, having easy access and decreasing the sampling error compared to other methods [10]. Histological examination of the surgical specimen showed exacerbated chronic cholecystitis, the biopsy of the pancreas showed the presence of chronic pancreatitis with diffuse fibrosis.

The patient's hospitalisation was prolonged and complicated by the onset of acute pancreatitis after about $24 \mathrm{~h}$. The patient was then submitted to intensive medical therapy, and subsequent radiological seriated controls showed a moderate degree of pancreatic necrosis (30-50\% of pancreatic tissue) with a stage $C$ according to the classification of Balthazar CT.

Then (25 post-operative days) the clinical sepsis for the infection of these necrotic areas unresponsive to antibiotic therapy became further aggravated. So

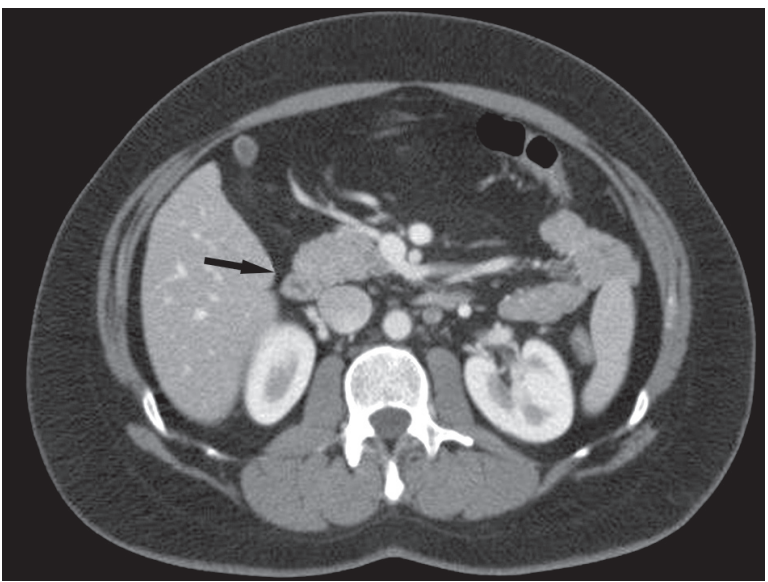

Figure 1. Pancreatic lesion (black arrow) a necrosectomy by was performed laparotomy with drainage of the abscess and placement of abdominal drainages (Figure 2).

\section{Discussion}

The difficulties in establishing a diagnosis are due to the lack of radiological signs about the Wirsung duct and the biliary tree and the lack of serological markers, probably influenced by the therapies that had already been followed by the patient for her autoimmune diseases. Because of that we needed to obtain a histological examination. This was done to avoid a duodeno-cephalo-pancreasectomy, which the patient could not tolerate. Recently, the international consensus on diagnostic criteria for AIP have been released, and the terms 'type 1 AIP' and 'type 2 AIP' have been introduced to describe the clinical profiles associated with lymphoplasmacytic sclerosing pancreatitis and idiopathic duct-centric pancreatitis. In the clinical setting, both type 1 and type 2 AIP have a similar presentation; they differ in demography, serology, involvement of other organs, and disease relapse rate. While type 1 is associated with the elevation of nonspecific autoantibodies and serum lgG4 levels, type 2 does not have definitive serological autoimmune markers. While most experts agree that the entities should be referred to as type 1 and type 2 AIP, respectively, others have concerns regarding the use of the term 'autoimmune' to describe idiopathic duct-centric pancreatitis [11]. According to five cardinal features of international consensus diagnostic criteria (ICDC) for AIP, namely imaging of pancreatic parenchyma and duct, serology, other organ involvement, pancreatic histology, and an optional criterion of response to steroid therapy [12], it was almost impossible to make a correct diagnosis, and the histological examination, even showing the findings related to the disease, was

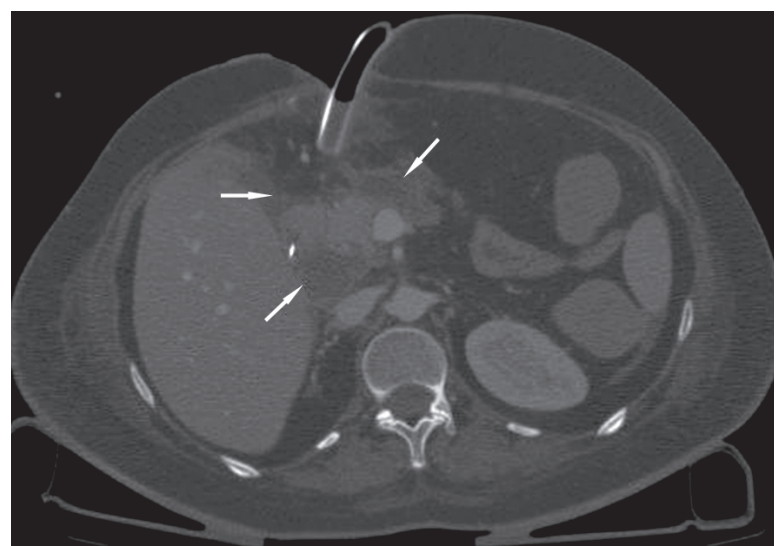

Figure 2. Pancreatic necrotic abscess with drainage (abscess identified by white arrows) 
not particularly diriment for the absence of the lymphocytic infiltrate ( $T$ lymphocytes) that characterises the disease in the active phase [10]. It was also probably "softened" by steroid therapy. From a surgical point of view, a wider resection (although that would have provided additional information on the lesion, in case it could be a tumour forming chronic pancreatitis [13]) cannot be indicated, and in our specific clinical case executable for the patient's conditions [14]. The surgical approach, although feasible with the appropriate indications and tricks [4], is an act oversized for diagnosis and represents the "extrema ratio".

\section{Conclusions}

The definitive diagnosis of autoimmune pancreatitis is very difficult because it requires the coexistence of several findings (radiological, serological, histological) and it is not always documented or even documentable. In patients with a medical history positive for autoimmune disorders who come to our attention with recurrent upper abdominal pain of an unclear nature or a nonspecific enlargement of the pancreas, possible AIP should be considered.

\section{Conflict of interest}

The authors declare no conflict of interest.

\section{References}

1. Ketikoglou I, Moulakakis A. Autoimmune pancreatitis. Dig Liver Dis 2005; 37: 211-5.

2. Sarles H, Sarles JC, Muratore R, Guien C. Chronic inflammatory sclerosis of the pancreas - an autonomous pancreatic disease? Am J Dig Dis 1961; 6: 688-98.

3. Suda K, Takase M, Fukumura Y, Kashiwagi SJ. Pathology of autoimmune pancreatitis and tumor-forming pancreatitis. J Gastroenterol 2007; 42 (Suppl. XVIII): 22-7.

4. Yeo CJ. Autoimmune sclerosing pancreatitis: the surgeon's perspective. J Gastrointest Surg 2005; 9: 11-4.

5. Gelrud A, Freedman SD. Autoimmune pancreatitis. J Gastrointest Surg 2005; 9: 1.

6. Okazaki K. Autoimmune pancreatitis is increasing in Japan. Gastroenterology 2003; 125: 1557-8.

7. Okazaki K, Kawa S, Kamisawa T, et al. Clinical diagnostic criteria of autoimmune pancreatitis: revised proposal. J Gastroenterology 2006; 41: 626-31.

8. Japan Pancreas Society. Diagnostic criteria for autoimmune pancreatitis. J Japan Pancreas Soc 2002; 17: 585-7.

9. Greenberger NJ. Autoimmune pancreatitis: time for a collective effort. Gastrointest Endosc 2007; 66: 1152-3.

10. Suda K, Takase M, Fukumura Y, et al. Can histopathology be the "gold standard" for diagnosing autoimmune pancreatitis? Gastroenterology 2005; 129: 2118-20.

11. Chari ST, Kloeppel G, Zhang L, et al. Histopathologic and clinical subtypes of autoimmune pancreatitis: the Honolulu consensus document. Pancreatology 2010; 10: 664-72.
12. Shimosegawa T, Chari ST, Frulloni L, et al. International consensus diagnostic criteria for autoimmune pancreatitis: guidelines of the international association of pancreatology. Pancreas 2011; 40: 352-8.

13. Koichi S, Masaru T, Yuki F, Satoko K. Pathology of autoimmune pancreatitis and tumor-forming pancreatitis. J Gastroenterol 2007; 42 (Suppl. XVIII): 22-7.

14. Cirocchi R, Trastulli S, Desiderio J, et al. Minimally invasive necrosectomy versus conventional surgery in the treatment of infected pancreatic necrosis: a systematic review and a meta-analysis of comparative studies. Surg Laparosc Endosc Percutan Tech 2013; 23: 8-20.

Received: 27.10 .2012

Accepted: 22.03 .2013 